\title{
Comparison of sex hormonal and metabolic profiles between omnivores and vegetarians in pre- and post-menopausal women
}

\author{
Antony D. Karelis ${ }^{1,2} *$, Annie Fex ${ }^{1}$, Marie-Eve Filion ${ }^{1}$, Herman Adlercreutz ${ }^{3}$ \\ and Mylène Aubertin-Leheudre ${ }^{1,2,3}$ \\ ${ }^{1}$ Department of Kinanthropology, Université du Québec à Montréal, Montreal, Canada \\ ${ }^{2}$ Institut Universitaire de Gériatrie de Montréal, Montreal, Canada \\ ${ }^{3}$ Folkhälsan Research Center, Institute for Preventive Medicine, Nutrition and Cancer, and Division of Clinical Chemistry, \\ University of Helsinki, Helsinki, Finland
}

(Received 30 November 2009 - Revised 4 February 2010 - Accepted 5 February 2010 - First published online 9 March 2010)

\begin{abstract}
The purpose of the present study was to investigate the sex hormonal and metabolic profiles in vegetarians and compare these with the profiles in omnivores. The design of the present study was cross-sectional. The study sample of pre- and post-menopausal women included forty-one omnivores and twenty-one vegetarians. Thereafter we determined: (1) plasma sex hormones, (2) fasting insulin, NEFA as well as apo-A and apo-B, (3) BMI, (4) a dietary profile (3 d dietary records), (5) physical activity and (6) total faecal excretion per $72 \mathrm{~h}$ and total urinary excretion per $72 \mathrm{~h}$. Vegetarians showed higher levels of sex hormone-binding globulin (SHBG), apo-A, total faecal excretion per $72 \mathrm{~h}$ and total fibre intake as well as lower levels of apo-B, free oestradiol, free testosterone, dehydroepiandrosterone sulfate (DHEA-s) and BMI. Interestingly, after controlling for BMI, significant differences between groups still persisted except for apo-B. Moreover, stepwise regression analysis showed that total fibre intake explained $15.2 \%$ of the variation in SHBG in our cohort, which accounted for the greatest source of unique variance. Results of the present study indicate that pre- and post-menopausal vegetarians present higher concentrations of SHBG, which could be explained, in part, by higher levels of fibre intake. This may explain, at least in part, the lower risk of developing type 2 diabetes.
\end{abstract}

Sex hormones: Dietary profiles: Sex hormone-binding globulin: Menopause: Total fibre

The diet of vegetarians is generally based on cereals, nuts, fibre, fruits and vegetables and may also include dairy products and eggs ${ }^{(1)}$. Several studies have reported that vegetarians are associated with having a favourable lipid profile as evidenced by lower levels of TAG, total cholesterol and LDL-cholesterol $^{(2-4)}$. In addition, vegetarians are associated with having lower levels of insulin resistance, intramyocellular lipids, blood pressure, BMI and C-reactive protein and higher levels of antioxidants ${ }^{(5-7)}$. Collectively, this favourable health profile may explain the lower risk of developing type 2 diabetes and CVD in vegetarians ${ }^{(2,8,9)}$.

Sex hormones have also been observed to be associated with metabolic complications. Higher levels of testosterone, oestradiol (E2) and dehydroepiandrosterone sulfate (DHEA-s) as well as lower levels of sex hormone-binding globulin (SHBG) have been shown to be associated with the metabolic syndrome and insulin resistance ${ }^{(10-13)}$. Furthermore, it had been reported that sex hormones may predict the development of type 2 diabetes $^{(13-15)}$. For example, a recent study showed that lower concentrations of SHBG could strongly predict the risk of type 2 diabetes $^{(15)}$ in men and women. Kalyani et al. ${ }^{(13)}$ indicated that higher levels of testosterone and E2 were associated with the development of type 2 diabetes in post-menopausal women.

Taken together, the lower risk of developing CVD and diabetes in vegetarians may be explained, at least in part, by a favourable sex hormone profile. However, the possible link between the protective profile of vegetarians with sex hormones and metabolic risk factors as well as the risk of type 2 diabetes and CVD is poorly understood. In fact, no data appear to be currently available on the inter-relationships between vegetarians, metabolic risk factors, sex hormones and the risk of developing chronic diseases (i.e. type 2 diabetes). Knowledge of a relationship between vegetarians with sex hormones may help us better understand the risk of disease of an individual and as such provide useful information to health professionals. Therefore, the purpose of the present study was to investigate the sex hormonal and metabolic profiles in vegetarians and compare these with the profiles in omnivores. We hypothesised that vegetarians would present a more favourable sex hormonal and metabolic profile compared with omnivores.

Abbreviations: DHEA-s, dehydroepiandrosterone sulfate; E2, oestradiol; SHBG, sex hormone-binding globulin.

* Corresponding author: Dr Antony Karelis, fax +1 514987 6616, email Karelis.antony@uqam.ca 


\section{Methods}

\section{Subjects}

We recruited two groups of women (forty-one omnivores and twenty-one vegetarians) living in the Helsinki area. The participants were voluntarily recruited by newspaper advertisements. Groups in the present study were age-matched. Based on their answers to a questionnaire about the frequency and the type of their physical activity, these women were sedentary or moderately physically active $(\leq 3 \mathrm{~h} /$ week with moderate intensity or $\leq 5 \mathrm{~h} /$ week with low intensity). We excluded subjects with a history of any major diseases such as type 2 diabetes and CVD or using drugs such as oral contraceptives, hormonal replacement therapy, or antibiotics. To be included as a regular vegetarian, the women were required to follow this diet for at least 2 years (mean 12 years). Sample blood collections for premenopausal women were performed during the mid-follicular phase of the menstrual cycle (days 5 to 9). The mean day of the menstrual cycle in which the blood collections began was on day $7 \pm 1$ for the omnivores and day $8 \pm 1$ for the vegetarians. The present study was conducted according to the guidelines laid down in the Declaration of Helsinki and all procedures involving human subjects were approved by the ethical committee of the Helsinki University Central Hospital. Subjects were initially interviewed by a doctor who explained the study and written informed consent was obtained from all subjects.

\section{Hormones}

Plasma samples were collected from fasting subjects. To the heparin plasma samples, we added $0.1 \%$ sodium azide and $0.1 \%$ ascorbic acid, and the samples were then frozen at $-20^{\circ} \mathrm{C}$ until analysed. As previously described, plasma oestrone (E1) and E2 were determined by RIA after chromatographic separation on an LH-20 column ${ }^{(16,17)}$. Plasma testosterone and androstenedione were determined as previously described by Kuoppasalmi et $a l^{(18)}$. SHBG was determined as described by Rosner ${ }^{(19)}$, with slight modifications ${ }^{(20)}$. Free E2 (in percentages and in pmol/l) was calculated from total E2 and SHBG values, and free testosterone (in percentages and in pmol/l) was calculated from testosterone and SHBG values according to Bergink et al. ${ }^{(21)}$. DHEA-s was measured by RIA with a commercial kit (Wien Laboratories, Succasunna, NJ, USA). Growth hormone, insulin, apo-A and apo-B were also measured by RIA with commercial kits (CIS International, Gif-sur-Yvette, France). All the methods have been validated with regard to accuracy, sensitivity, specificity and reproducibility.

\section{Total faecal excretion}

The total amount of faecal excretion was collected during a $72 \mathrm{~h}$ period, kept cold and brought daily to the laboratory by the subject.

\section{Dietary intake}

Each subject was provided with a food scale balance and was instructed on how to complete the dietary records. The validity of a $3 \mathrm{~d}$ dietary record to estimate dietary intake in adults without cognitive impairments has been confirmed ${ }^{(22)}$. Dietary analyses were completed by a nutritionist using the 1983 version of a coding system from the Department of Nutrition (University of Helsinki) for total, lipid, carbohydrate, protein, cholesterol, MUFA, PUFA, starch, sucrose, lactose, Ca and alcohol intake. For fibre data, we used Southgate tables $^{(23)}$, and for some typical Finnish foods (i.e. rye products), we used the data given by manufacturers. Fibre values are presented separately for cereal, vegetables, berries/fruits and total.

\section{Physical activity level}

The level of physical activity was evaluated with a frequency questionnaire (how long and how many sessions per week). We categorised women as physically inactive if they practised physical activity three or fewer times or $\leq 3 \mathrm{~h} / \mathrm{week}$. The level of physical activity has been considered low or moderate when the intensity was between 0 and $8 \mathrm{kcal} / \mathrm{min}(0-33 \mathrm{~kJ} / \mathrm{min})$ or less than $1000 \mathrm{kcal} /$ week $(4184 \mathrm{~kJ} /$ week $)$.

\section{Statistical analysis}

Data are expressed as the mean values and standard deviations. An independent $t$ test was used for the comparison of variables between omnivores and vegetarians as well as between pre- and post-menopausal vegetarian women. Moreover, using a univariate general linear model analysis, we statistically controlled for BMI to verify if it would abolish differences in SHBG, free E2, free testosterone, DHEA-s, total faecal excretion per $72 \mathrm{~h}$, apo-B and apo-A between groups. A $\chi^{2}$ test was used to compare menopause status between the two groups. Pearson correlations were performed to examine the relationship between SHGB with free E2, free testosterone, DHEA-s, total faecal excretion per $72 \mathrm{~h}$, apo-B apo-A, BMI, total fibre and muscle mass. Finally, a stepwise multi-linear regression analysis was performed to identify predictors of SHBG. Independent variables considered in the final model for SHBG were total fibre, BMI, apo-A, apo-B, total faecal excretion per $72 \mathrm{~h}$ and free E2. We chose to determine factors predicting SHBG because a recent study showed that lower concentrations of SHBG could strongly predict the risk of type 2 diabetes in men and women ${ }^{(15)}$. Therefore, we find it timely to examine predictors of SHBG. Statistical analysis was performed using SPSS 15 for Windows (SPSS, Inc. Chicago, IL, USA). Significance was accepted at $P<0.05$.

\section{Results}

Physical and metabolic characteristics of omnivores and vegetarians are presented in Table 1. Both groups were comparable for age, menopause status, age of menopause, height, physical activity, insulin, NEFA and total urinary excretion per $72 \mathrm{~h}$. Body weight, BMI, apo-B, and the apo-B:apo-A ratio were significantly lower in vegetarians $(P<0 \cdot 05)$, whereas apo-A and total faecal excretion per $72 \mathrm{~h}$ were significantly higher in vegetarians $(P<0 \cdot 01)$.

Plasma steroid hormones are shown in Table 2. SHBG was significantly higher in vegetarians $(P<0.01)$, whereas free $\mathrm{E} 2$, free testosterone and DHEA-s were significantly lower $(P<0.05)$. No other differences were noted. 
Table 1. Physical and metabolic characteristics of omnivores and vegetarians (Mean values and standard deviations)

\begin{tabular}{|c|c|c|c|c|c|}
\hline \multirow[b]{2}{*}{ Variables } & \multicolumn{2}{|c|}{ Omnivores ( $n$ 41) } & \multicolumn{2}{|c|}{ Vegetarians (n 21) } & \multirow[b]{2}{*}{$P$} \\
\hline & Mean & SD & Mean & SD & \\
\hline Age (years) & $47 \cdot 0$ & 14.9 & $47 \cdot 7$ & $13 \cdot 2$ & NS \\
\hline Menopause status (\%) & & & & & NS \\
\hline Height $(\mathrm{cm})$ & 164 & 5.9 & 164 & 5.9 & NS \\
\hline Body weight (kg) & 63.9 & $9 \cdot 2$ & $58 \cdot 7^{*}$ & 7.9 & 0.029 \\
\hline $\operatorname{BMI}\left(\mathrm{kg} / \mathrm{m}^{2}\right)$ & $23 \cdot 8$ & $3 \cdot 2$ & $21 \cdot 7^{\star}$ & 2.5 & 0.015 \\
\hline Physically active (\%) & & & & & NS \\
\hline Insulin $(\mu \mathrm{U} / \mathrm{l})$ & $8 \cdot 0$ & $5 \cdot 0$ & $7 \cdot 3$ & $2 \cdot 6$ & NS \\
\hline $\operatorname{NEFA}(\mathrm{mmol} / \mathrm{l})$ & 0.56 & 0.2 & 0.59 & 0.2 & NS \\
\hline apo-B (g/l) & 0.83 & 0.3 & $0.69^{*}$ & 0.2 & 0.038 \\
\hline apo-A (g/l) & 1.44 & 0.2 & $1.60^{*}$ & 0.2 & 0.009 \\
\hline apo-B:apo-A & 0.60 & 0.2 & $0.44^{*}$ & 0.1 & 0.004 \\
\hline Total faecal excretion $(\mathrm{g} / 72 \mathrm{~h})$ & $379 \cdot 2$ & 131 & $528 \cdot 7^{*}$ & 192 & 0.001 \\
\hline Total urinary excretion $(\mathrm{ml} / 72 \mathrm{~h})$ & 4002 & 1181 & 4539 & 2022 & NS \\
\hline
\end{tabular}

${ }^{\star}$ Mean value was significantly different from that of omnivores $(P<0 \cdot 05)$.

Dietary characteristics of omnivores and vegetarians are described in Table 3. Energy intake was comparable between both groups. Carbohydrates, total fibre, vegetable fibre, berry/ fruit fibre and total fibre/kg body weight were significantly higher in vegetarians, whereas protein, total fat, MUFA and cholesterol were significantly lower in vegetarians. No other differences were noted.

When statistically controlling for BMI, significant differences in SHBG, total faecal excretion per $72 \mathrm{~h}$, apo-A, free $\mathrm{E} 2$, free testosterone and DHEA-s levels between the groups persisted. When the apo-B:apo-A ratio and apo-B levels were statistically controlled for BMI, statistical significance was abolished between the groups. Furthermore, significant correlations were observed between SHBG with total faecal excretion per $72 \mathrm{~h}$, free $\mathrm{E} 2$, free testosterone, apo-A, apo-B, apo-B:apo-A ratio and total fibre.

In a sub-analysis, using all vegetarians in our cohort ( $n 21)$, we compared the hormonal, metabolic and dietary profiles of pre- ( $n$ 11) and post- ( $n$ 10) menopausal women separately (using Mann-Whitney analysis). Results show that no differences were observed for hormonal (except for oestrogen-related hormones), metabolic and dietary profiles between pre- and post-menopausal vegetarian women. However, the present results should be considered preliminary due to the low number of subjects, but they may hopefully stimulate interest in the need for greater participant characterisation in research protocols. Moreover, we analysed the sex hormone levels in the plasma for pre- ( $n$ 22) and post-menopausal (n 19) omnivores and found that oestrone, E2, free E2, androstenedione, SHBG, DHEA-s and DHEA levels were significantly lower in post-menopausal omnivores. In addition, no differences in testosterone and free testosterone as well as growth hormone were observed between the groups. It should be noted that the age of menopause for vegetarians and omnivores was 50 (SD 2) v. 50 (SD 4) years, respectively, and the age of the postmenopausal women at the start of the study for vegetarians and omnivores was 60 (SD 4) v. 61 (SD 7) years, respectively. Therefore, the mean length of menopause for vegetarians and omnivores was 10 v. 11 years, respectively (NS).

Finally, we performed a stepwise regression analysis to identify independent predictors of SHBG. Table 4 illustrates the summary of the model. The present results show that total fibre and BMI were independent predictors of SHBG, collectively explaining $25.0 \%$ of the variance $(P<0.01)$.

Table 2. Plasma steroid hormones in omnivores and vegetarians

(Mean values and standard deviations)

\begin{tabular}{|c|c|c|c|c|c|}
\hline \multirow[b]{2}{*}{ Variables } & \multicolumn{2}{|c|}{ Omnivores ( $n$ 41) } & \multicolumn{2}{|c|}{ Vegetarians ( $n$ 21) } & \multirow[b]{2}{*}{$P$} \\
\hline & Mean & SD & Mean & SD & \\
\hline Oestrone (pmol/l) & $211 \cdot 7$ & 78 & $227 \cdot 8$ & 116 & NS \\
\hline Oestradiol (pmol//) & $153 \cdot 6$ & 82 & $155 \cdot 7$ & 118 & NS \\
\hline Testosterone (nmol//) & 2.09 & 1.32 & 1.77 & 0.6 & NS \\
\hline $\mathrm{SHBG}(\mathrm{nmol} / \mathrm{l})$ & $44 \cdot 8$ & $16 \cdot 9$ & $70 \cdot 2^{*}$ & $18 \cdot 7$ & $<0.001$ \\
\hline Growth hormone $(\mu \mathrm{g} / \mathrm{l})$ & $3 \cdot 22$ & 2.5 & 3.62 & 2.5 & NS \\
\hline Free oestradiol (\%) & $2 \cdot 12$ & $0 \cdot 1$ & $1.99^{\star}$ & 0.1 & 0.001 \\
\hline Free oestradiol $(\mathrm{pmol} / \mathrm{l})$ & $3 \cdot 24$ & $1 \cdot 7$ & 3.02 & $2 \cdot 3$ & NS \\
\hline Free testosterone (\%) & 1.00 & 0.2 & $0.88^{*}$ & 0.1 & 0.007 \\
\hline Free testosterone $(\mathrm{pmol} / \mathrm{l})$ & $18 \cdot 3$ & $6 \cdot 1$ & $15 \cdot 5$ & $5 \cdot 6$ & NS \\
\hline Androstenedione (nmol/l) & 4.82 & 1.8 & $4 \cdot 84$ & $2 \cdot 1$ & NS \\
\hline DHEA sulfate $(\mu \mathrm{mol} / \mathrm{l})$ & $6 \cdot 31$ & $5 \cdot 0$ & $3 \cdot 68^{\star}$ & $2 \cdot 4$ & 0.026 \\
\hline DHEA $(\mathrm{nmol} / \mathrm{l})$ & $18 \cdot 3$ & $10 \cdot 2$ & $18 \cdot 6$ & 9.5 & NS \\
\hline
\end{tabular}

SHBG, sex hormone-binding-globulin; DHEA, dehydroepiandrosterone.

${ }^{*}$ Mean value was significantly different from that of omnivores $(P<0.05)$. 
Table 3. Dietary characteristics of omnivores and vegetarians

(Mean values and standard deviations)

\begin{tabular}{|c|c|c|c|c|c|}
\hline \multirow[b]{2}{*}{ Variables } & \multicolumn{2}{|c|}{ Omnivores $(n 41)$} & \multicolumn{2}{|c|}{ Vegetarians (n 21) } & \multirow[b]{2}{*}{$P$} \\
\hline & Mean & SD & Mean & SD & \\
\hline \multicolumn{6}{|l|}{ Energy intake } \\
\hline $\mathrm{MJ} / \mathrm{d}$ & $7 \cdot 66$ & 1.4 & 7.62 & 1.4 & NS \\
\hline $\mathrm{kcal} / \mathrm{d}$ & 1832 & 333 & 1823 & 323 & NS \\
\hline Carbohydrates (\% energy) & $60 \cdot 0$ & $4 \cdot 1$ & $64.9^{*}$ & 3.9 & $<0.001$ \\
\hline Protein (\% energy) & $18 \cdot 7$ & $2 \cdot 2$ & $16 \cdot 0^{*}$ & 1.9 & $<0.001$ \\
\hline Total fat (\% energy) & $21 \cdot 3$ & $2 \cdot 8$ & $19 \cdot 1^{*}$ & $2 \cdot 9$ & 0.005 \\
\hline Saturated fat $(g / d)$ & $40 \cdot 0$ & 10 & 34.6 & 10 & 0.052 \\
\hline Monounsaturated fat $(\mathrm{g} / \mathrm{d})$ & $25 \cdot 4$ & $6 \cdot 2$ & $21 \cdot 5^{\star}$ & $5 \cdot 2$ & 0.017 \\
\hline Polyunsaturated fat $(\mathrm{g} / \mathrm{d})$ & $9 \cdot 8$ & 3.4 & $70 \cdot 6$ & 273 & NS \\
\hline Cholesterol (mg/d) & 380 & 95 & $243^{*}$ & 79 & $<0.001$ \\
\hline Total fibre $(\mathrm{g} / \mathrm{d})$ & $16 \cdot 8$ & 4.4 & $23 \cdot 7^{*}$ & 7.5 & $<0.001$ \\
\hline Cereal fibre $(\mathrm{g} / \mathrm{d})$ & $9 \cdot 3$ & $2 \cdot 7$ & $10 \cdot 9$ & $3 \cdot 3$ & 0.053 \\
\hline Vegetable fibre $(\mathrm{g} / \mathrm{d})$ & 3.4 & 1.5 & $5 \cdot 8^{*}$ & $2 \cdot 7$ & $<0.001$ \\
\hline Berry/fruit fibre (g/d) & $2 \cdot 7$ & $2 \cdot 2$ & $5 \cdot 2^{*}$ & 4.8 & 0.008 \\
\hline Total fibre per kg body weight $(\mathrm{g} / \mathrm{d})$ & 0.27 & 0.1 & $0.41^{*}$ & 0.2 & $<0.001$ \\
\hline
\end{tabular}

${ }^{*}$ Mean value was significantly different from that of omnivores $(P<0.05)$.

\section{Discussion}

Several studies have reported that vegetarians are associated with a favourable metabolic profile, which could reduce the risk of developing type 2 diabetes and $\mathrm{CVD}^{(2,6,9)}$. To add to the body of literature, we attempted to provide new information on metabolic risk factors such as sex hormones that may characterise the profile of vegetarians in pre- and postmenopausal women.

We hypothesised that vegetarians would present a more favourable sex hormonal and metabolic profile compared with omnivores. Results from the present study support our hypothesis. That is, we found that SHBG levels were significantly higher by $56.7 \%$ in vegetarian women compared with omnivore women. Higher concentrations of SHBG have been recently shown to be associated with a favourable metabolic profile as well as reducing the risk of type 2 diabetes ${ }^{(11,15)}$. In addition, vegetarians had lower levels of free E2, free testosterone and DHEA-s. Furthermore, vegetarians showed higher levels of apo-A, total faecal excretion per $72 \mathrm{~h}$ and total fibre intake as well as lower levels of apo-B, BMI and total fat intake. Moreover, several studies have shown that higher fibre intake may be associated with higher total faecal excretion mass and a more favourable metabolic profile ${ }^{(24-26)}$. In the present study we observed higher levels of total faecal excretion per $72 \mathrm{~h}$ and total fibre intake in vegetarians. Interestingly, after controlling for BMI, all metabolic and hormonal differences between the groups remained significant. This suggests that the differences between the groups were independent of BMI. However, differences in apo-A levels between the groups were abolished after controlling for BMI, suggesting that BMI may be a potential mediating factor.
Collectively, these results suggest that vegetarians display favourable metabolic, sex hormonal and dietary profiles compared with omnivores in our cohort, which may be associated with a decreased risk of type 2 diabetes and CVD. These findings confirm and extend the results of several other studies, which also showed that vegetarians are associated with a favourable metabolic and dietary profile ${ }^{(2,4,6,11,27)}$. These results also suggest that multiple factors could be implicated in the healthy profile of vegetarian women. Therefore, studies characterising vegetarians may want to consider several outcome measures in different domains in order to have a complete understanding of vegetarians.

In a sub-analysis, using all vegetarians in our cohort, we compared the hormonal, metabolic and dietary profiles of pre- and post-menopausal women separately. Interestingly, results in the present study show that no differences were observed for hormonal (except for oestrogen-related hormones), metabolic and dietary profiles between pre- and post-menopausal vegetarian women. This suggests that the protective profile of vegetarians could be sustained after the menopause. This finding is important since the menopause could be associated with an increased risk of $\mathrm{CVD}^{(28,29)}$.

Results from the logistic regression analysis showed that total fibre intake and BMI were independent predictors of SHBG in our cohort. This suggests that higher levels of total fibre intake and a lower BMI may be associated with higher levels of SHBG. In support of this idea, we showed that total fibre intake explained $15.2 \%$ of the variation in SHBG levels in our cohort, which accounted for the greatest source of unique variance. Therefore, high fibre intake could contribute to high levels of SHBG, which may lead to a lower risk of

Table 4. Stepwise regression analysis regarding independent predictors of sex hormone-binding globulin (SHBG)

\begin{tabular}{|c|c|c|c|c|c|}
\hline Dependent variable & Step & Independent variable & Partial $r^{2}$ & Total $r^{2}$ cumulative & $P$ \\
\hline \multirow[t]{2}{*}{ SHBG $(\mathrm{nmol} / \mathrm{l})$} & 1 & Total fibre & 0.152 & 0.152 & 0.002 \\
\hline & 2 & BMI & 0.098 & 0.250 & 0.008 \\
\hline
\end{tabular}


developing type 2 diabetes. This would be a testable hypothesis that warrants further investigation.

The present study has several limitations. First, our cohort is only composed of non-diabetic pre- and post-menopausal women. Therefore, our findings are limited to this population. Second, we used a cross-sectional approach, which does not allow us to conclude any causal associations between SHBG levels and total fibre intake in our cohort. Finally, the present study lacks detailed body composition measures and dietary intake may be under- and mis-reported. Nonetheless, the present results are strengthened by studying a well-characterised cohort.

In conclusion, results of the present study indicate that preand post-menopausal vegetarians present favourable hormonal, metabolic and dietary profiles, in particular, higher concentrations of SHBG, which may be associated with higher levels of total fibre intake. This favourable dietary, hormonal and metabolic profile may explain, at least in part, the lower risk of developing type 2 diabetes in vegetarians.

\section{Acknowledgements}

The present study was supported by the Medical Research Council in the Academy of Finland, the Sigrid Jusélius Foundation and Samfundet Folkhälsan. M. A.-L. was supported by the Canadian Institutes of Health Research (CIHR). A. D. K. was supported by the Fonds de la Recherche en Santé du Québec (FRSQ).

A. D. K. contributed to the analysis and interpretation of data as well as to the writing of the manuscript. A. F. and M.-E. F. were involved in the writing of the manuscript and provided significant advice. H. A. designed the experiment and provided significant advice. M. A.-L. was involved in the collection of data and provided significant advice and revisions.

The authors report no conflict of interest.

\section{References}

1. Dwyer JT (1988) Health aspects of vegetarian diets. Am J Clin Nutr 48, 712-738.

2. Key TJ, Davey GK \& Appleby PN (1999) Health benefits of a vegetarian diet. Proc Nutr Soc 58, 271-275.

3. Li D, Ball M, Bartlett M, et al. (1999) Lipoprotein(a), essential fatty acid status and lipoprotein lipids in female Australian vegetarians. Clin Sci (Lond) 97, 175-181.

4. Lee HY, Woo J, Chen ZY, et al. (2000) Serum fatty acid, lipid profile and dietary intake of Hong Kong Chinese omnivores and vegetarians. Eur J Clin Nutr 54, 768-773.

5. Szeto YT, Kwok TC \& Benzie IF (2004) Effects of a long-term vegetarian diet on biomarkers of antioxidant status and cardiovascular disease risk. Nutrition 20, 863-866.

6. Goff LM, Bell JD, So PW, et al. (2005) Veganism and its relationship with insulin resistance and intramyocellular lipid. Eur J Clin Nutr 59, 291-298.

7. Valachovicova M, Krajcovicova-Kudlackova M, Blazicek P, et al. (2006) No evidence of insulin resistance in normal weight vegetarians. A case control study. Eur J Nutr 45, 52-54.

8. Fraser GE (2009) Vegetarian diets: what do we know of their effects on common chronic diseases? Am J Clin Nutr 89. $1607 \mathrm{~S}-1612 \mathrm{~S}$

9. Tonstad S, Butler T, Yan R, et al. (2009) Type of vegetarian diet, body weight, and prevalence of type 2 diabetes. Diabetes Care 32, 791-796.
10. Polderman KH, Gooren LJ, Asscheman H, et al. (1994) Induction of insulin resistance by androgens and estrogens. J Clin Endocrinol Metab 79, 265-271.

11. Onat A, Hergenc G, Karabulut A, et al. (2007) Serum sex hormone-binding globulin, a determinant of cardiometabolic disorders independent of abdominal obesity and insulin resistance in elderly men and women. Metabolism 56, 1356-1362.

12. Ibanez L, Lopez-Bermejo A, Diaz M, et al. (2009) Low-birth weight children develop lower sex hormone binding globulin and higher dehydroepiandrosterone sulfate levels and aggravate their visceral adiposity and hypoadiponectinemia between six and eight years of age. J Clin Endocrinol Metab 94, 3696-3699.

13. Kalyani R, Franco M, Dobs AS, et al. (2009) The association of endogenous sex hormones, adiposity, and insulin resistance with incident diabetes in postmenopausal women. $J$ Clin Endocrinol Metab 94, 4127-4135.

14. Ding EL, Song Y, Manson JE, et al. (2007) Plasma sex steroid hormones and risk of developing type 2 diabetes in women: a prospective study. Diabetologia 50, 2076-2084.

15. Ding EL, Song Y, Manson JE, et al. (2009) Sex hormonebinding globulin and risk of type 2 diabetes in women and men. $N$ Engl J Med 361, 1152-1163.

16. Adlercreutz H, Fotsis T \& Heikkinen R (1982) Current state of the art in the analysis of estrogens. In Advances in Steroid Analyses, pp. 3-33. Budapest: Ahadémia Kiado.

17. Goldin BR, Adlercreutz H, Gorbach SL, et al. (1982) Estrogen excretion patterns and plasma levels in vegetarian and omnivorous women. $N$ Engl J Med 307, 1542-1547.

18. Kuoppasalmi K, Naveri H, Rehunen S, et al. (1976) Effect of strenuous anaerobic running exercise on plasma growth hormone, cortisol, luteinizing hormone, testosterone, androstenedione, estrone and estradiol. J Steroid Biochem 7, 823-829.

19. Rosner W (1972) A simplified method for the quantitative determination of testosterone-estradiol-binding globulin activity in human plasma. J Clin Endocrinol Metab 34, 983-988.

20. Kuoppasalmi K (1980) Plasma testosterone and sex-hormonebinding globulin capacity in physical exercise. Scand J Clin Lab Invest 40, 411-418.

21. Bergink EW, Holma P \& Pyorala T (1981) Effects of oral contraceptive combinations containing levonorgestrel or desogestrel on serum proteins and androgen binding. Scand J Clin Lab Invest 41, 663-668.

22. Luhrmann PM, Herbert BM, Gaster C, et al. (1999) Validation of a self-administered 3-day estimated dietary record for use in the elderly. Eur J Nutr 38, 235-240.

23. Paul AA \& Southgate DA (1979) McCance and Widdowson's 'The Composition of Foods': dietary fibre in egg, meat and fish dishes. J Hum Nutr 33, 335-336.

24. Jenkins DJ, Kendall CW, Popovich DG, et al. (2001) Effect of a very-high-fiber vegetable, fruit, and nut diet on serum lipids and colonic function. Metabolism 50, 494-503.

25. Li J, Kaneko T, Qin LQ, et al. (2003) Effects of barley intake on glucose tolerance, lipid metabolism, and bowel function in women. Nutrition 19, 926-929.

26. McIntosh GH, Noakes M, Royle PJ, et al. (2003) Whole-grain rye and wheat foods and markers of bowel health in overweight middle-aged men. Am J Clin Nutr 77, 967-974.

27. Laaksonen DE, Niskanen L, Punnonen K, et al. (2004) Testosterone and sex hormone-binding globulin predict the metabolic syndrome and diabetes in middle-aged men. Diabetes Care 27, $1036-1041$.

28. Gorodeski GI (2002) Update on cardiovascular disease in postmenopausal women. Best Pract Res Clin Obstet Gynaecol 16, 329-355.

29. Knopp RH (2002) Risk factors for coronary artery disease in women. Am J Cardiol 89, 28E-35E. 